\title{
COMMENTARY
}

\section{Extravascular lung water in acute respiratory distress syndrome: potential clinical value, assumptions and limitations}

\author{
Luigi Camporota*, Mark De Neef and Richard Beale \\ See related research by Chew et al., http://ccforum.com/content/16/1/R1
}

\begin{abstract}
Extravascular lung water has been proposed as a marker of disease severity, response to treatment and mortality in patients with acute respiratory distress syndrome. Here, we discuss its potential value and limitations in clinical practice.
\end{abstract}

Acute respiratory distress syndrome (ARDS) is characterised by diffuse alveolar damage and increased extravascular lung water (EVLW), clinically measured by the trans-pulmonary single-indicator thermo-dilution method. Although not part of the diagnostic criteria for ARDS, increased EVLW is central to the pathophysiology of the syndrome and correlates with the severity of lung injury, response to treatment and clinical outcome. In this issue of Critical Care, Chew and colleagues [1], in an observational study involving an heterogeneous population of patients with shock, report the role of EVLW in the context of two themes: its ability to predict disease severity and outcome and its added value as a diagnostic criterion of ARDS.

On the first theme, Chew and colleagues [1] report that patients with higher EVLW indices (EVLWIs) had more severe disease with greater Lung Injury Score, and were less likely to survive ICU [1]. These findings are in accordance with the available literature [2-4], which suggests that EVLW is a good predictor of mortality with an odds ratio of 8.8 [5]. However, there is still no firm consensus on its range of normal values and the best indexing parameter (for example, actual or predicted body weight

*Correspondence: luigi.camporota@gstt.nhs.uk

Department of Adult Critical Care - Guy's and St Thomas' NHS Foundation Trust, St Thomas' Hospital, 1st Floor East Wing, Lambeth Palace Road, London, SE1 7EH, United Kingdom or pulmonary blood volume) against which EVLW should be normalised. Chew and colleagues [1] found that EVLW indexed to absolute body weight resulted in a stronger association with outcome. This result differs from previous clinical studies showing that EVLW indexed to predicted body weight $(\mathrm{PBW})$ is a superior predictor of mortality [6], of developing multiple organ failure [2] and for the diagnosis of ARDS [4,6-8]. The physiologic rationale for using predicted body weight is that lung size and lung weight depend on height and gender - and therefore predicted body weight - whereas adjusting EVLW for absolute body weight may lead to erroneous estimations of EVLW in obese patients. However, obesity may be an explanatory variable rather than a confounding one. Obesity may directly affect EVLW - for example, by decreasing the intra-thoracic blood volume (ITBV) or the lymphatic clearance of EVLW through increased intra-abdominal and pleural pressures. As EVLW is calculated as the difference between the intrathoracic thermal volume and ITBV, any change in pulmonary perfusion and ITBV will affect EVLW.

In addition, the relationship between EVLW and disease severity, and EVLW and outcome, may reflect three independent but concurrent possibilities.

First, EVLWI may measure the amount of inflammatory oedema (high permeability). The ratio of EVLW to the pulmonary blood volume has been used as an indirect index of pulmonary vascular permeability in critically ill patients with, or at risk of, ARDS [9], with normal ratios helping to exclude high pulmonary permeability [10]. Chew and colleagues [1] show that this ratio seems a better marker of disease severity in patients with a Lung Injury Score $>2.5$, perhaps implying that patients with severe ARDS have greater lung oedema, possibly due to greater pulmonary permeability, and greater mortality. Computed tomography studies of patients with ARDS have suggested that patients with severe ARDS have greater inflammatory oedema and lung weight, a greater response to lung recruitment and higher positive 
end-expiratory pressure (PEEP) together with higher mortality [11]. Considering the reported limitations of this index to truly distinguish high permeability from hydrostatic oedema [9], however, this relationship is here only suggested and warrants further clinical confirmation.

Second, EVLWI may reflect a higher fluid balance (hydrostatic pulmonary oedema), which is an independent determinant of mortality and is associated with worse oxygenation and lower compliance [12]. The lack of correlation with other oxygenation parameters and compliance suggests that EVLW may provide additional information not available in other parameters.

Third, EVLW may be a reflection of other physiological processes that are associated with disease severity but independent from oedema formation (linked to the technique). Pathologic factors leading to an increase in dead space, shunt fraction and loss of hypoxic pulmonary vasoconstriction in severe ARDS cause redistribution of pulmonary blood flow in parallel with the distribution of the perfused thermal volume. Since the thermal signal can only diffuse into perfused lung tissue, the increased perfusion of previously thermally silent tissue causes an apparent increase of EVLW independently of true changes in pulmonary oedema [13].

On the second theme of the inclusion of EVLWI as a diagnostic criterion of ARDS, the current definition of ARDS has been challenged on the basis of its limited predictive value, large inter-observer variability and the lack, among its defining criteria, of an objective physiological marker that possesses diagnostic, prognostic and concurrent validity (that is, the ability to distinguish ARDS from other forms of hypoxaemic respiratory failure). Elevated EVLWI can identify patients with ARDS and predicts progression to ARDS in patients with risk factors 2 to 3 days before the patients meet current ARDS criteria, increasing the opportunity of delivering therapeutic intervention proven to improve outcome [14]. Chew and colleagues [1] show that EVLW used as an additional diagnostic test leads to a two- to three-fold increase in the post-test odds ratio for the diagnosis of ARDS, and the authors argue for its inclusion in a revised definition of ARDS. This argument is justified in view of the clear pathophysiological and prognostic plausibility of EVLW in ARDS $[2-4,8,15,16]$. In our opinion, however, physiological and methodological assumptions required for its estimation, the still arbitrary cutoff values and the still limited availability worldwide currently restrict its general applicability as a diagnostic criterion in the definition of ARDS until its prognostic and concurrent validity are prospectively validated and confirmed in larger scale clinical trials.

\section{Abbreviations}

ARDS, acute respiratory distress syndrome; EVLW, extravascular lung water: EVLWI, extravascular lung water index; ITBV, intra-thoracic blood volume.

\section{Competing interests}

The authors declare that they have no competing interests.

Published: 27 February 2012

\section{References}

1. Chew MS, Ihrman L, During J, Bergenzaun L, Ersson A, Unden J, Ryden J, Akerman E, Larsson M: Extravascular lung water index improves the diagnostic accuracy of lung injury in patients with shock. Crit Care 2012, 16:R1.

2. Chung FT, Lin HC, Kuo CH, Yu CT, Chou CL, Lee KY, Kuo HP, Lin SM: Extravascular lung water correlates multiorgan dysfunction syndrome and mortality in sepsis. PLoS One 2010, 5:e15265.

3. Effros RM, Pornsuriyasak P, Porszasz J, Casaburi R: Indicator dilution measurements of extravascular lung water: basic assumptions and observations. Am J Physiol Lung Cell Mol Physiol 2008, 294:L1023-L1031 .

4. Maharaj R: Extravascular lung water and acute lung injury. Cardiol Res Pract 2012, 2012:407035.

5. Zhang Z, Lu B, Ni H: Prognostic value of extravascular lung water index in critically ill patients: A systematic review of the literature. J Crit Care 2011, [Epub ahead of print].

6. Craig TR, Duffy MJ, Shyamsundar M, McDowell C, McLaughlin B, Elborn JS, McAuley DF: Extravascular lung water indexed to predicted body weight is a novel predictor of intensive care unit mortality in patients with acute lung injury. Crit Care Med 2010, 38:114-120.

7. Berkowitz DM, Danai PA, Eaton S, Moss M, Martin GS: Accurate characterization of extravascular lung water in acute respiratory distress syndrome. Crit Care Med 2008, 36:1803-1809.

8. Brown LM, Liu KD, Matthay MA: Measurement of extravascular lung water using the single indicator method in patients: research and potential clinical value. Am J Physiol Lung Cell Mol Physiol 2009, 297:L547-L558.

9. Groeneveld $A B$, Verheij J: Extravascular lung water to blood volume ratios as measures of permeability in sepsis-induced ALI/ARDS. Intensive Care Med 2006, 32:1315-1321.

10. van der Heijden M, Groeneveld AB: Extravascular lung water to blood volume ratios as measures of pulmonary capillary permeability in nonseptic critically ill patients. J Crit Care 2010, 25:16-22.

11. Gattinoni L, Caironi P, Cressoni M, Chiumello D, Ranieri VM, Quintel M, Russo S, Patroniti N, Cornejo R, Bugedo G: Lung recruitment in patients with the acute respiratory distress syndrome. N Engl J Med 2006, 354:1775-1786.

12. Rosenberg AL, Dechert RE, Park PK, Bartlett RH, NIH NHLBI ARDS Network: Review of a large clinical series: association of cumulative fluid balance on outcome in acute lung injury: a retrospective review of the ARDSnet tidal volume study cohort. I Intensive Care Med 2009, 24:35-46.

13. Easley RB, Mulreany DG, Lancaster CT, Custer JW, Fernandez-Bustamante A Colantuoni E, Simon BA: Redistribution of pulmonary blood flow impacts thermodilution-based extravascular lung water measurements in a model of acute lung injury. Anesthesiology 2009, 111:1065-1074.

14. Letourneau JL, Pinney J, Phillips CR: Extravascular lung water predicts progression to acute lung injury in patients with increased risk. Crit Care Med 2011 [Epub ahead of print].

15. Michard F: Bedside assessment of extravascular lung water by dilution methods: temptations and pitfalls. Crit Care Med 2007, 35:1 186-1192.

16. Sakka G: Prognostic value of extravascular lung water in critically ill patients. Chest 2002, 122:2080-2086.

doi:10.1186/cc11187

Cite this article as: Camporota $L$, et al:: Extravascular lung water in acute respiratory distress syndrome: potential clinical value, assumptions and limitations. Critical Care 2012, 16:114. 